Preprint

UCRL-JC-137794

\title{
Low-spin collective excitations in deformed nuclei: When is a $\beta$ a $\beta$ vibration?
}

\author{
P.E. Garrett
}

This article was submitted to $10^{\text {th }}$ International Symposium on Capture Gamma Ray Spectroscopy and Related Topics, Santa Fe, NM, August 29September 3, 1999

February 24, 2000

U.S. Department of Energy

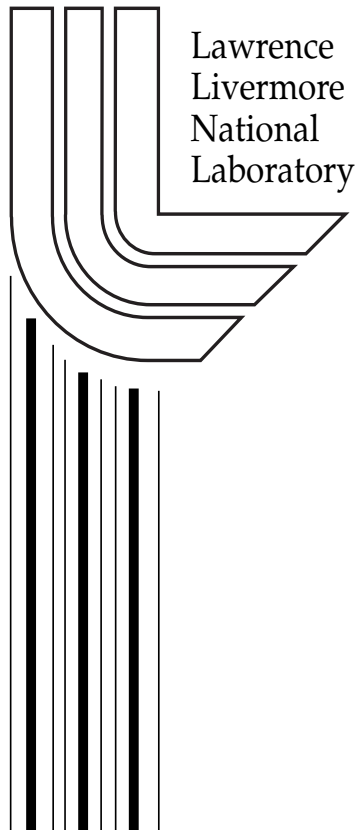




\section{DISCLAIMER}

This document was prepared as an account of work sponsored by an agency of the United States Government. Neither the United States Government nor the University of California nor any of their employees, makes any warranty, express or implied, or assumes any legal liability or responsibility for the accuracy, completeness, or usefulness of any information, apparatus, product, or process disclosed, or represents that its use would not infringe privately owned rights. Reference herein to any specific commercial product, process, or service by trade name, trademark, manufacturer, or otherwise, does not necessarily constitute or imply its endorsement, recommendation, or favoring by the United States Government or the University of California. The views and opinions of authors expressed herein do not necessarily state or reflect those of the United States Government or the University of California, and shall not be used for advertising or product endorsement purposes.

This is a preprint of a paper intended for publication in a journal or proceedings. Since changes may be made before publication, this preprint is made available with the understanding that it will not be cited or reproduced without the permission of the author.

This report has been reproduced

directly from the best available copy.

Available to DOE and DOE contractors from the

Office of Scientific and Technical Information

P.O. Box 62, Oak Ridge, TN 37831

Prices available from (423) 576-8401

http://apollo.osti.gov/bridge/

Available to the public from the

National Technical Information Service

U.S. Department of Commerce

5285 Port Royal Rd.,

Springfield, VA 22161

http://www.ntis.gov/

OR

Lawrence Livermore National Laboratory

Technical Information Department's Digital Library

http://www.llnl.gov/tid/Library.html 


\title{
Low-spin collective excitations in deformed nuclei: When is a $\beta$ a $\beta$ vibration?
}

\author{
P.E. Garrett \\ Lawrence Livermore National Laboratory, Livermore, CA 94551 USA
}

\begin{abstract}
The $0_{2}^{+}$level in deformed nuclei has historically been given the label $\beta$, even though its properties vary drastically from one nucleus to another. By reviewing models from where the original term $\beta$ vibration arose, and appealing to microscopic calculations, guidelines are proposed for the properties of a true $\beta$ vibration. An examination of available data, including $B(E 2)$ values, $\rho^{2}(E 0)$ values, and single- and two-nucleon-transfer populations, shows that very few $0_{2}^{+}$levels satisfy the criteria for a $\beta$ vibration. It is suggested that pairing excitations may be playing a dominant role.
\end{abstract}

\section{INTRODUCTION}

The existence of a $\beta$-vibrational mode arose naturally as a consequence of the quantization of the Hamiltonian for a deformed liquid drop with surface vibrations [1]. Since the $K^{\pi}=2^{+} \gamma$-vibrational mode was observed as the lowest-lying $2^{+}$ excitation, it was naturally expected that the lowest-lying excited $0^{+}$state would be the $\beta$-vibrational excitation. As low-lying excited $0^{+}$states in a wide range of deformed nuclei were observed, they were interpreted as $\beta$-vibrational states. Higher-lying $0^{+}$states were often interpreted as two-quasiparticle excitations. With the development of techniques for extracting absolute $B(E 2)$ values, especially

Coulomb excitation, it was found that the $B\left(E 2 ; 0_{\mathrm{gs}}^{+} \rightarrow 2_{0_{2}^{+}}^{+}\right)$values displayed a wide variation over well-deformed nuclei, with variations of orders of magnitude over a single isotopic chain. The continued practice of labeling the $0_{2}^{+}$state by " $\beta$ ", irrespective of its properties, has reduced the value of the term, and the original definitions $[1,2]$ of the properties of a $\beta$ vibration have been lost.

\section{MACROSCOPIC CONSIDERATIONS}

In order to give guidelines as to how a $\beta$ vibration should behave, it is necessary to consider its origins. Like the $\gamma$ vibration, the $\beta$ mode arose from quantizing the Hamiltonian of the macroscopic liquid drop. Within the context of the rotationvibration model, which is based on th Bohr-Mottelson Hamiltonian (see Ref. [2] and references therein), the expressions for $B(E 2)$ values are 


$$
\begin{aligned}
B\left(E 2 ; I_{\mathrm{i}}^{\mathrm{gsb}} \rightarrow I_{\mathrm{f}}^{\mathrm{gsb}}\right) & =\left(\frac{3 Z R_{\circ}^{2}}{4 \pi}\right)^{2} \frac{2 I_{\mathrm{f}}+1}{2 I_{\mathrm{i}}+1} \beta_{\circ}^{2}\left(I_{\mathrm{f}} 020 \mid I_{\mathrm{i}} 0\right)^{2}(1+\alpha)^{2} \\
B\left(E 2 ; I_{\gamma} \rightarrow I_{\mathrm{gsb}}\right) & =\left(\frac{3 Z R_{\circ}^{2}}{4 \pi}\right)^{2} \frac{2 I_{\mathrm{gsb}}+1}{2 I_{\gamma}+1} \beta_{\circ}^{2}\left(I_{\mathrm{gsb}} 022 \mid I_{\gamma} 2\right)^{2} x^{2}(1-2 \alpha)^{2} \\
B\left(E 2 ; I_{\beta} \rightarrow I_{\mathrm{gsb}}\right) & =\left(\frac{3 Z R_{\circ}^{2}}{4 \pi}\right)^{2} \frac{2 I_{\mathrm{gsb}}+1}{2 I_{\beta}+1} \beta_{\circ}^{2}\left(I_{\mathrm{gsb}} 020 \mid I_{\beta} 0\right)^{2} y^{2}(1+2 \alpha)^{2}
\end{aligned}
$$

where

$$
\alpha=\frac{2}{7} \sqrt{\frac{5}{\pi}} \beta_{\circ}, \quad x=\sqrt{\frac{3 \hbar^{2}}{\mathcal{J}_{\circ} E_{\gamma}}}, \quad y=\sqrt{\frac{3 \hbar^{2}}{2 \mathcal{J}_{\circ} E_{\beta}}} .
$$

Taking ratios of $B(E 2)$ values results in

$$
\frac{B\left(E 2 ; 0_{\beta}^{+} \rightarrow 2_{\mathrm{gsb}}^{+}\right)}{B\left(E 2 ; 2_{\mathrm{gsb}}^{+} \rightarrow 0_{\mathrm{gs}}^{+}\right)}=5 \frac{y^{2}(1+2 \alpha)^{2}}{(1+\alpha)^{2}}, \quad \frac{B\left(E 2 ; 2_{\gamma}^{+} \rightarrow 0_{\mathrm{gs}}^{+}\right)}{B\left(E 2 ; 2_{\mathrm{gsb}}^{+} \rightarrow 0_{\mathrm{gs}}^{+}\right)}=\frac{x^{2}(1-2 \alpha)^{2}}{(1+\alpha)^{2}} .
$$

Assuming a typical rotational parameter of $15 \mathrm{keV}$, a deformation $\beta_{\circ}=0.25$, a $\beta$-vibrational energy of $1.2 \mathrm{MeV}$ (a typical energy of the first excited $0^{+}$state), a $\gamma$-vibrational energy of $0.8 \mathrm{MeV}$, and a $2_{\mathrm{gsb}}^{+} \rightarrow 0_{\mathrm{gs}}^{+} B(E 2)$ value of $150 \mathrm{~W} . \mathrm{u}$., these expressions yield $B\left(E 2 ; 2_{\gamma}^{+} \rightarrow 0_{\text {gs }}^{+}\right)=9.5 \mathrm{~W}$.u. and $B\left(E 2 ; 0_{\beta}^{+} \rightarrow 2_{\text {gsb }}^{+}\right)=33$ W.u. Also, the $B(E 2)$ value for the decay of the $2^{+}$member of the $0^{+}$bands (which are the ones known in most cases) should have $B\left(E 2 ; 2_{\beta}^{+} \rightarrow 0_{\mathrm{gs}}^{+}\right)=6.6 \mathrm{~W}$.u. The prediction for the $\gamma$-to-ground $B(E 2)$ value is slightly higher than usually observed (typically $\simeq 5$ W.u.), but the model certainly gives a good guideline as to what one should expect the magnitude of the $\beta$-to-ground band transitions to be.

Another approach is to assert that the $\beta$ vibration have the same degree of collectivity as the $\gamma$ vibration. A manifestation of this assertion is that the intrinsic matrix elements be similar between the two types of quadrupole vibration. The $B(E 2)$ value is related to the intrinsic matrix element by the relation

$$
B(E 2)=\left(\left\langle I_{i} K_{i} 2 \Delta K \mid I_{f} K_{f}\right\rangle\left\langle I_{f}\|E 2\| I_{i}\right\rangle\right)^{2}\left(1+\delta_{K_{1}=0 \neq K_{2}}\right) .
$$

and using the $B\left(E 2 ; 2_{\gamma}^{+} \rightarrow 0_{\mathrm{gs}}^{+}\right)$value of $5 \mathrm{~W} . \mathrm{u}$., as is typical in the deformed rareearth region, the $B\left(E 2 ; 0_{\beta}^{+} \rightarrow 2_{\text {gsb }}^{+}\right)$value should be on the order of 12 W.u. In a similar manner, the $B\left(E 2 ; 2_{\beta}^{+} \rightarrow 0_{\mathrm{gs}}^{+}\right)$values should be on the order of $2.5 \mathrm{~W} . \mathrm{u}$. for the band to be considered as a $\beta$-vibrational candidate. However, this rather small value for the $B\left(E 2 ; 2_{\beta}^{+} \rightarrow 0_{\mathrm{gs}}^{+}\right)$transition must be used with great care, and possible mixing effects must be considered.

Shown in Fig.1 are the experimentally known $B\left(E 2 ; 0_{2}^{+} \rightarrow 2_{\text {gsb }}^{+}\right)$(top panel) and $B\left(E 2 ; 0_{\mathrm{gs}}^{+} \rightarrow 2_{0_{2}^{+}}^{+}\right)$(bottom panel) values. The broad bands indicate the range 

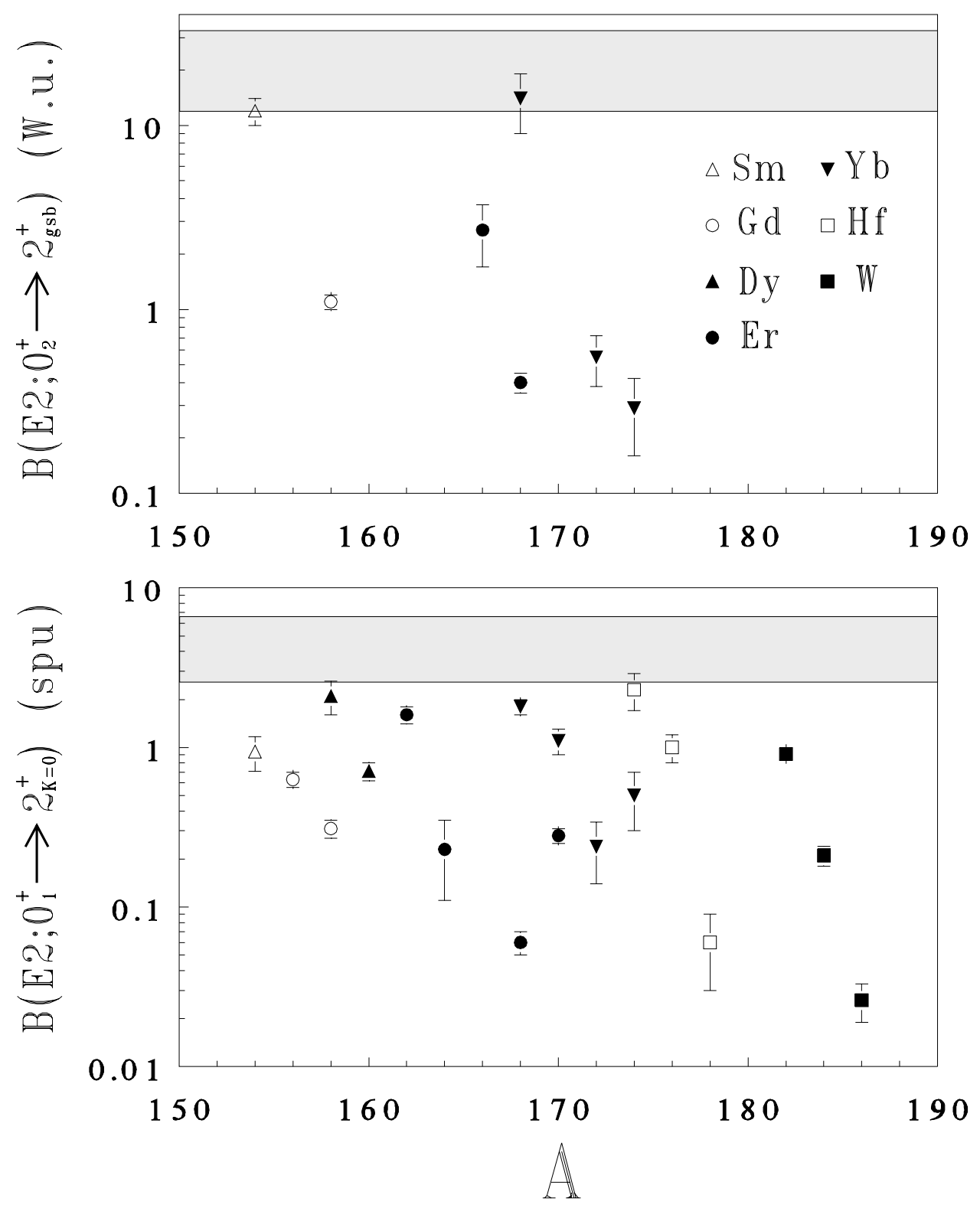

FIGURE 1. $B(E 2)$ values for the $0_{2}^{+} \rightarrow 2_{\text {gsb }}^{+}$transition in W.u. (top panel), as well as the known $B\left(E 2 ; 0_{\mathrm{gs}}^{+} \rightarrow 2_{0_{2}^{+}}^{+}\right)$values in single-particle units (bottom panel). The horizontal bands shown on the figure are the expected values for a true $\beta$ vibration, as described in the text. The candidates for $0_{2}^{+}$levels as $\beta$ vibrations are limited to ${ }^{154} \mathrm{Sm}$ and ${ }^{168} \mathrm{Yb}$ from the $B\left(E 2 ; 0_{2}^{+} \rightarrow 2_{\text {gsb }}^{+}\right)$data, and ${ }^{158} \mathrm{Dy},{ }^{174} \mathrm{Hf}$ and possibly ${ }^{162} \mathrm{Er},{ }^{168} \mathrm{Yb}$ from the $B\left(E 2 ; 0_{\mathrm{gs}}^{+} \rightarrow 2_{0_{2}^{+}}^{+}\right)$data.

expected for a good $\beta$-vibrational candidate. As can be seen, only in a few cases do the $B(E 2)$ values indicate the possibility of significant $\beta$-vibrational strength.

The $\beta$ vibration should also give rise to enhanced $E 0$ transitions due to the radial shape oscillations in $\beta$. The expression for $\rho^{2}(E 0)$ is [3] 


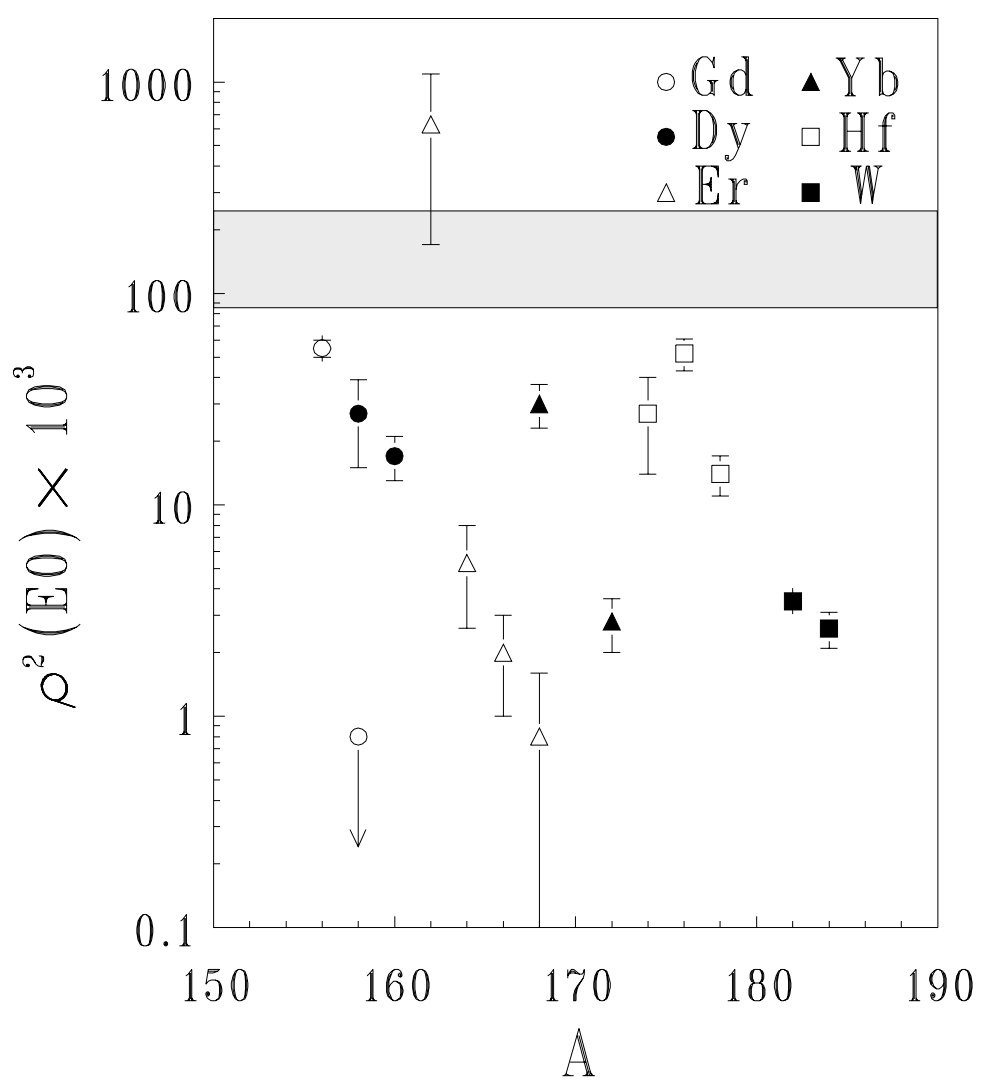

FIGURE 2. Values of $\rho^{2}(E 0)$ for the first excited $0^{+}$band from the compilation of Wood et al. [3] for well-deformed rare-earth nuclei. The horizontal band indicates the range of $\rho^{2}(E 0)$ values one would expect for good $\beta$ vibrations based on the assumption of a $B\left(E 2 ; 0_{\beta}^{+} \rightarrow 2_{\text {gsb }}^{+}\right)$of $12-33$ W.u. Only in ${ }^{162} \operatorname{Er}$ has a value been measured which corresponds to the expected value for a $\beta$ vibration. The large value for ${ }^{162}$ Er, however, has been questioned by the authors of Ref. [3].

$$
\rho^{2}\left(E 0 ; n_{\beta}=1 \rightarrow n_{\beta}=0\right)=\frac{B\left(E 2 ; 0_{\mathrm{gs}}^{+} \rightarrow 2_{\beta}^{+}\right) 4 \beta_{0}^{2}}{e^{2} r_{0}^{4} A^{4 / 3}}
$$

where $r_{0}=1.2 \mathrm{fm}$ and $B(E 2)$ is in units of $e^{2} \mathrm{fm}^{4}$. There is no angular momentum dependence in the $\rho^{2}$ values, so that the $0_{\beta}^{+} \rightarrow 0_{\mathrm{gs}}^{+}, 2_{\beta}^{+} \rightarrow 2_{\mathrm{gsb}}^{+}$, etc., values are identical. Using the aforementioned $B\left(E 2 ; 0_{\beta}^{+} \rightarrow 2_{\text {gsb }}^{+}\right)$value range of $12-33$ W.u., one expects a $\rho^{2}(E 0) \times 10^{3}$ value of 85 to 230 to characterize a valid $\beta$-vibrational candidate. Figure 2 shows the $\rho^{2}(E 0)$ values compiled by Wood et al. [3], together with the "expected" range for a true $\beta$ vibration.

\section{MICROSCOPIC CONSIDERATIONS}

There are two main contributions to the wave functions of excited $0^{+}$states; those forming $K^{\pi}=0^{+} \lambda \bar{\lambda}$ pairs and those forming $K^{\pi}=0^{+} \lambda \mu$ pairs where $\mu \neq \bar{\lambda}$. 

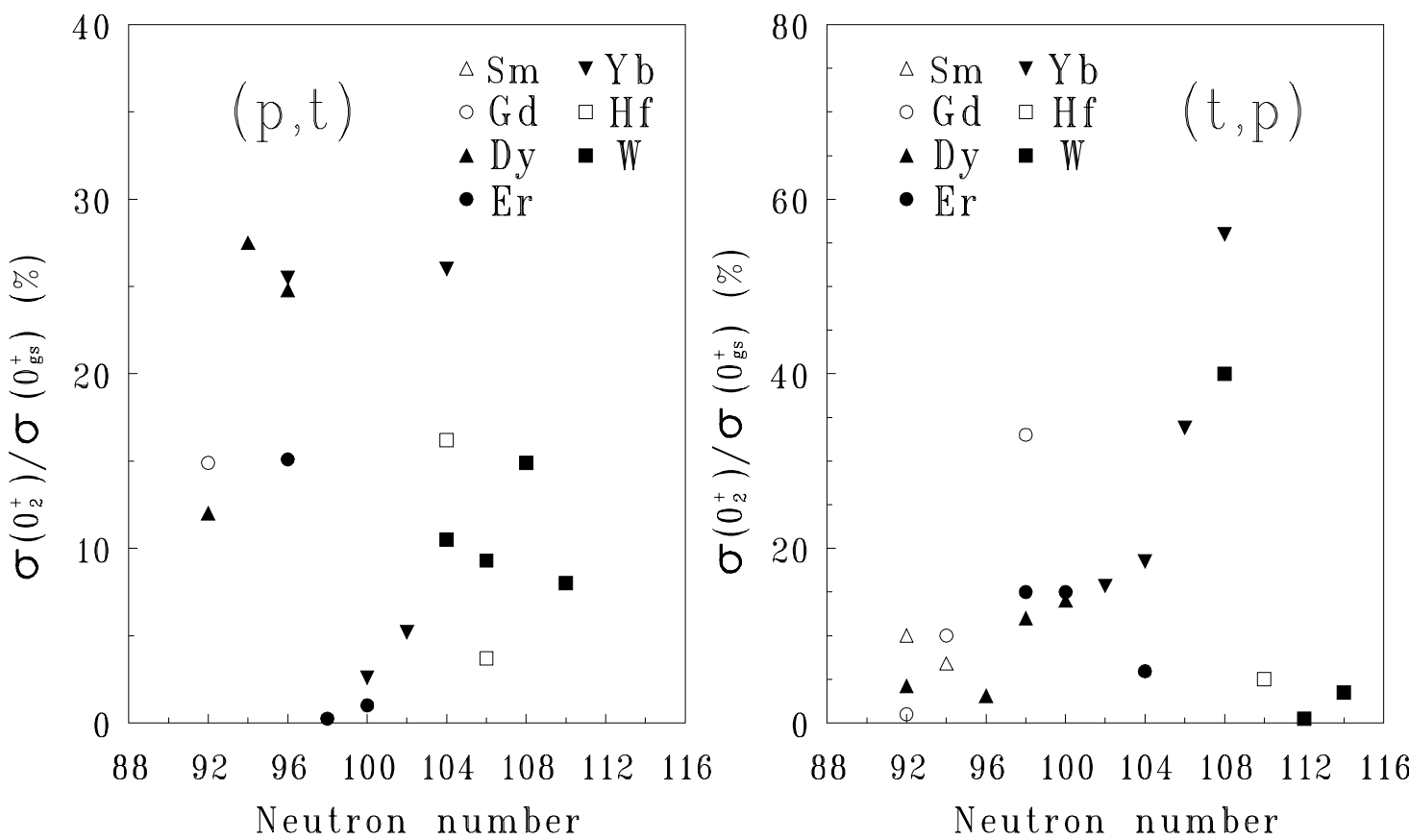

FIGURE 3. Ratio of two-neutron-transfer cross sections for the first excited state to that of the ground state. The data are corrected for $Q$-value effects by DWBA calculations and are plotted as a function of neutron number for the final nucleus. Significant population of an excited $0^{+}$ state implies that the wave function has a significant amplitude of $\lambda \bar{\lambda}$ terms. For ${ }^{160} \mathrm{Gd}(N=96)$, ${ }^{160} \mathrm{Dy}(N=98)$, and ${ }^{180} \mathrm{Hf}(N=108)$, the $0_{2}^{+}$levels were not observed in the $(t, p)$ reactions, while in ${ }^{158} \mathrm{Gd}(N=94),{ }^{162} \mathrm{Dy}(N=96),{ }^{168} \mathrm{Yb}(N=98)$, and ${ }^{178} \mathrm{Hf}(N=106)$, the $0_{2}^{+}$levels were not observed in the $(p, t)$ reactions.

The $\lambda \bar{\lambda}$ pairs are pairs of quasiparticles in time-reversed orbits, and dominate when proper account is taken of the various terms in the Hamiltonian [4]. Zawischa, Speth and $\mathrm{Pal}[5]$ argue that, in analogy to vibrational states in spherical nuclei, a true $\beta$ vibration consists of a superposition of a large number of generalized $\mathrm{p}-\mathrm{h}(\lambda \mu$ with $\mu \neq \bar{\lambda}$ ) states with comparable amplitudes. Pairing should have no decisive effect on a $\beta$ vibration, but rather should give rise to collective excitations consisting of a superposition of $\lambda \bar{\lambda}$ pairs [5]. The calculations of Ref. [5] revealed that the true $\beta$ mode occurs at very high energies, in the giant resonance region, and all low-lying $0^{+}$states (below twice the pairing gap) are of the pairing type.

Wave functions for $K^{\pi}=0^{+}$states that are dominated by $\lambda \bar{\lambda}$ terms may be populated strongly in two-nucleon-transfer reactions. The transfer of a pair of nucleons coupled to $J=0, L=0$ involves $\lambda \bar{\lambda}$ pairs located close to the Fermi surface. Thus, two-nucleon transfer probes the microscopic components in the wave function which are of the form $\lambda \bar{\lambda}$. As is well known, the ground states of well-deformed nuclei are populated strongly in $(p, t)$ and $(t, p)$ reactions due to the large overlap of the wave functions between the $N$ and $N \pm 2$ system when the pair creation or annihilation operator is applied. For strong population of an excited 
$0^{+}$state, this state must also be composed of many terms in the wave function of similar form as the ground state.

Shown in Fig.3 are the two-neutron-transfer cross sections, corrected for $Q$-value dependence by DWBA calculations, for population of the $0_{2}^{+}$state relative to that of the ground state. As can be seen, there are wide variations in the cross section to the $0_{2}^{+}$level, but in many cases there are strong populations amounting to greater than $10 \%$ of the ground state strength. This implies that for the $(t, p)$ reaction there must be significant terms in the $0_{2}^{+}$state wave function involving $\lambda \bar{\lambda}$ terms from above the Fermi surface, whereas for the $(p, t)$ reaction these terms must involve orbitals from below the Fermi surface. It should be noted that two-neutrontransfer does not address the issue of proton pairs. Furthermore, the absence of strong population does not necessarily mean the absence of $\lambda \bar{\lambda}$ terms in the wave function.

As in two-nucleon-transfer reactions, single-nucleon-transfer reactions sample the microscopic details of the wave functions. In many cases where single-nucleontransfer reactions have been performed, the excited $0^{+}$bands are not observed. The non-population of an excited $0^{+}$band only implies that it does not contain a significant amplitude of $\{$ target configuration $\} \otimes\{$ transferred configuration $\}$ and does not address the nature of the band. However, in those cases where an excited $0^{+}$band is strongly populated, some definite statements can be made. The only cases known to the author where a $0^{+}$band has been observed have involved those where the $\{$ transferred configuration $\}=\{$ target configuration $\}$, i.e., populating components in the final state wave function of the form $\lambda \bar{\lambda}$. Some specific examples of this are in ${ }^{178} \mathrm{Hf}[6]$ and ${ }^{172} \mathrm{Yb}[7]$. In ${ }^{178} \mathrm{Hf}$, the $(p, \alpha)$ reaction populated the $0_{2}^{+}$ band stronger than the ground state band. It was concluded [6] that the $0_{2}^{+}$band had a dominant component of the $7 / 2^{+}[404]-7 / 2^{+}[404]$ two-quasiproton configuration. In ${ }^{172} \mathrm{Yb}[7]$, the $(d, t)$ and $(t, d)$ reactions populated the $0_{2}^{+}$band with $53 \%$ and $9 \%$ of the strength of the ground state band, respectively, indicating significant amplitudes of the $5 / 2^{-}[512]-5 / 2^{-}[512]$ and $1 / 2^{-}[521]-1 / 2^{-}[521]$ two-quasineutron configurations in the $0_{2}^{+}$wave function. Large $\lambda \bar{\lambda}$ components in the wave functions of excited $0^{+}$levels are not expected for a pure $\beta$-vibrational state, and are more in line with what is expected for a pairing excitation or a two-quasiparticle state.

\section{IMPLICATIONS}

When all data are viewed, it becomes clear that there are few good examples for the $0_{2}^{+}$level as a $\beta$-vibrational state. Only in ${ }^{154} \mathrm{Sm},{ }^{158} \mathrm{Dy},{ }^{162} \mathrm{Er},{ }^{168} \mathrm{Yb}$, and ${ }^{174} \mathrm{Hf}$, do the $B(E 2)$ values possibly indicate significant $\beta$-vibrational strength.

In ${ }^{154} \mathrm{Sm}$, the lifetime of the $0_{2}^{+}$level was recently measured by Krücken et al. [8], and a $B\left(E 2 ; 0_{2}^{+} \rightarrow 2_{\text {gsb }}^{+}\right)$value of $12(2)$ W.u. was determined. The population of this level in the $(t, p)$ reaction with $10 \%$ of the ground-state strength indicates appreciable $\lambda \bar{\lambda}$ components in its wave function. While this may be the best example to date of a $0_{2}^{+}$state having a $\beta$-vibrational character, it is probably not a 
pure $\beta$ vibration. For ${ }^{158}$ Dy, the $B(E 2)$ value of $2.1(5)$ s.p.u. for the excitation of the $2_{0_{2}^{+}}^{+}$level is one of the most collective $0_{\mathrm{gs}}^{+} \rightarrow 2_{0_{2}^{+}}^{+}$values measured. However, the $\rho^{2}(E 0)$ value of $27(12) \times 10^{-3}[3]$ is rather small, and it also receives a significant population in the $(p, t)$ reaction $(12 \%)$. In ${ }^{162} \mathrm{Er}$, the $B\left(E 2 ; 0_{\mathrm{gs}}^{+} \rightarrow 2_{0_{2}^{+}}^{+}\right)$value has a very large uncertainty $(100 \%)$ arising most from a large uncertainty in the lifetime measurement [9]. The same problem affects the large $\rho^{2}(E 0)$ value deduced, and it has been suggested [3] that the lifetime is considerably longer. For ${ }^{168} \mathrm{Yb}$, the $B(E 2)$ value of $1.8(2)$ s.p.u. [10] indicates some collectivity. The $B\left(E 2 ; 0_{2}^{+} \rightarrow 2_{\text {gsb }}^{+}\right)$ value has also been measured [10] to be $14(5) \mathrm{W}$.u. The $\rho^{2}(E 0)$ value of $30(7) \times 10^{-3}$ [3] is somewhat small, but the fact that it was not observed in the $(p, t)$ reaction and the enhanced $B(E 2)$ values make it an attractive candidate for a significant fraction of the $\beta$-vibrational strength. ${ }^{174} \mathrm{Hf}$ was the "classic" example of a $0_{2}^{+}$state as a $\beta$ vibration [1]. It has the largest $B\left(E 2 ; 0_{\mathrm{gs}}^{+} \rightarrow 2_{0_{2}^{+}}^{+}\right)$value in Fig.1, although its $\rho^{2}(E 0)$ value of $27(13) \times 10^{-3}$ [3] is small. Two-neutron-transfer data do not exist for this nucleus.

While it is clear that there are very few good examples of a $\beta$ vibration, the question arises as to the nature of the $0_{2}^{+}$states. In most cases, the energies of the $0_{2}^{+}$states are well-below twice the pairing gap energy, indicative of collective components in their wave functions. Thus, they are probably not predominately two-quasiparticle states, where various components in the wave function are admixed in a non-coherent fashion and do not display any collective behaviour. A $K^{\pi}=0^{+}$two-phonon interpretation has been suggested [11], but must await the measurement of enhanced $B(E 2)$ values for the transition from the $0_{2}^{+}$level to the $2_{\gamma}^{+}$state. The few measurements $[12,13]$ which exist to date of the absolute $B(E 2)$ value do not support such an interpretation. As alluded to earlier, the evidence would appear to favor an interpretation of the majority of $0_{2}^{+}$levels as pairing excitations. This interpretation has been advanced by Zawischa, Speth, and Pal [5]. Within the QPM, it also appears that the one-phonon states are pairing excitations, as they are described as being comprised of many two-quasiparticle components of the form $\lambda \bar{\lambda}$. The small $B(E 2)$ values for the excitation of the $2^{+}$members of the $0_{2}^{+}$band, and the large two-neutron-transfer strength observed in many nuclei are consistent with such an interpretation. In some cases, such as ${ }^{178} \mathrm{Hf}[6]$ and ${ }^{172} \mathrm{Yb}$ [7], the large cross sections observed in single-nucleon transfer also indicate wave functions dominated by specific $\lambda \bar{\lambda}$ components.

\section{SUMMARY}

A set of guidelines has been proposed for classifying the first excited $0^{+}$states in well-deformed rare-earth nuclei. For states to be properly labeled as a $\beta$ vibration, they should have: 1) $B\left(E 2 ; 0_{\beta}^{+} \rightarrow 2_{\mathrm{gs}}^{+}\right)$values of $12-33 \mathrm{~W} . u$. , or conversely $B\left(E 2 ; 2_{\beta}^{+} \rightarrow 0_{\mathrm{gs}}^{+}\right)$of $2.5-6.6$ W.u., 2) $\rho^{2}(E 0) \times 10^{3}$ values of $\left.85-230,3\right)$ "small" two-nucleon transfer strengths, 4) small single-nucleon transfer strengths for the 
situation where the transferred configuration is the time-reversed target configuration $(\lambda \bar{\lambda})$. Very few $K^{\pi}=0_{2}^{+}$states satisfy these criteria. The properties of the $\beta$ vibration presented here stem from the original definition of the state, and these properties must be followed in order that the term be useful in classifying nuclear states. It is from the macroscopic picture that the terminology $\beta$ vibration, or $\beta$ phonon, arose, and it is in the macroscopic picture that its definition must be anchored.

When all experimental data are examined, it is clear that, unlike the $\gamma$ vibrations, the properties of the $K^{\pi}=0_{2}^{+}$state depend on the changing Fermi surface to a great deal. It is suggested that this behaviour is more indicative of pairing-type excitations. This interpretation is favored when large strengths are observed to the $0_{2}^{+}$state in two-nucleon transfer reactions. That the low-lying $0^{+}$states may be dominated by pairing terms in their wave functions does not imply that these states are non-collective; they can be very collective with many terms in the wave function acting coherently. It should be remembered that $B(E 2)$ values are not the sole indicator of the degree of collectivity that a state possesses. In addition, excited $0_{2}^{+}$states may contain admixtures of $\beta$-vibrational, two-phonon, and two-

quasiparticle terms, and information from all experimental data must be considered before making assignments.

This work was performed under the auspices of the U.S. Department of Energy by Lawrence Livermore National Laboratory under DOE contract no. W-7405Eng-48.

\section{REFERENCES}

1. A. Bohr and B.R. Mottelson, Nuclear Structure, Vol. II, pub. W.A. Benjamin, Inc., Reading Mass. USA, 1975.

2. J.M. Eisenberg and W. Greiner, Nuclear Theory, Vol. I, pub. North Holland, Amsterdam, 1987.

3. J.L. Wood, E.F. Zganjar, C. De Coster, and K. Heyde, Nucl. Phys. A651, 323 (1999).

4. V.G. Soloviev, Nucl. Phys. 69, 1 (1965).

5. D. Zawischa, J. Speth, and D. Pal, Nucl. Phys. A311, 445 (1978).

6. D.G. Burke, O. Straume, G. Løvhøiden, T.F. Thorsteinsen, and A. Graue, Nucl. Phys. A569, 523 (1994).

7. D.G. Burke, V.G. Soloviev, A.V. Sushkov, and N. Yu. Shirikova, Nucl. Phys. A656, 287 (1999).

8. R. Krücken et al., Phys. Lett. B 454, 15 (1999).

9. R.M. Ronningen et al., Phys. Rev. C 26, 97 (1982).

10. L.L. Riedinger et al., Phys. Rev. C 20, 2170 (1979).

11. R.F. Casten and P. von Brentano, Phys. Rev. C 50, R1280 (1994).

12. P.E. Garrett et al., Phys. Lett. B400, 250 (1997).

13. T. Härtlein, M. Heinebrodt, D. Schwalm, and C. Fahlander, Eur. Phys. J. A 2, 253 (1998). 